\title{
ON PERTURBATION OF SCHRÖDINGER OPERATOR ON AXIS BY NARROW POTENTIALS
}

\author{
A.R. BIKMETOV, V.F. VIL'DANOVA, I.KH. KHUSNULLIN
}

\begin{abstract}
We consider a Schrödinger operator on the axis with two complex-valued potentials depending on two small parameters. One of these parameters describes the length of the supports of the potentials, while the other corresponds to the maximal values of the absolute values of the potentials. We obtain the sufficient condition ensuring the emergence of an eigenvalues from the threshold of the essential spectrum. The asymptotics for this eigenvalue is constructed.
\end{abstract}

Keywords: Schrödinger operator, perturbation, asymptotics.

Mathematics Subject Classification: 35J10, 34E10

\section{Problem AN MAIN RESUlts}

Let $V_{1}(x)$ and $V_{2}(x)$ be complex-valued functions in $C_{0}^{\infty}(\mathbb{R}), x_{1}, x_{2}$ be arbitrary numbers $\left(x_{1}<x_{2}\right)$, parameters $0<\mu, \varepsilon \ll 1$ satisfy the relation

$$
\varepsilon \mu^{-1}=o(1) \text {. }
$$

In the work we consider the operator

$$
\mathcal{H}_{\varepsilon, \mu}:=-\frac{d^{2}}{d x^{2}}+\mu^{-1}\left(V_{1}\left(\frac{x-x_{1}}{\varepsilon}\right)+V_{2}\left(\frac{x-x_{2}}{\varepsilon}\right)\right), \quad x_{1}<x_{2}
$$

in $L_{2}(\mathbb{R})$ on domain $W_{2}^{2}(\mathbb{R})$.

It is well-known (see, for instance, [1, Ch. V]) that operator $\mathcal{H}_{0}:=-\frac{d^{2}}{d x^{2}}$ in $L_{2}(\mathbb{R})$ on domain $W_{2}^{2}(\mathbb{R})$ is self-adjoint, its discrete spectrum is empty, and the continuous spectrum coincides with the semi-axis $[0,+\infty)$. Since functions $V_{j}$ are compactly supported, by [1, Ch. IV, Thms. 1.1, 5.35]) continuous spectrum of operator $\mathcal{H}_{\varepsilon, \mu}$ is the semi-axis $[0,+\infty$ ) (for the case of complex-valued functions see, for instance, [2]).

In [3] - 6] the effect of new eigenvalues emerging from the thresholds of the continuous spectrum for the operator $\mathcal{H}_{0}+\varepsilon W(x)$ was studied, where $W(x)$ is a real-valued potential decaying sufficiently fast at infinity. In [3] the case when function $W(x)$ satisfies the condition $\int_{\mathbb{R}}|W(x)|\left(1+x^{2}\right) d x<\infty$ was considered. It was shown that if $\int_{\mathbb{R}} W(t) d t \leqslant 0$, operator $\mathcal{H}_{0}+\varepsilon W(x)$ had the unique eigenvalue. In [4] a wider class of functions $W(x)$ was considered, for which $\int_{\mathbb{R}}|W(x)|\left(1+x^{2}\right) d x=\infty$. Three cases were treated when $W(x)$ behaves as $-a x^{-\beta}$ for $x \rightarrow \infty$. It was shown that as $2<\beta<3$, the results obtained in [3] remained true. In [5] there was proven the existence of the eigenvalue for the operator $\mathcal{H}_{0}+\varepsilon W(x)$, when $W(x)$

A.R. Bikmetov, V.F. Vil'danova, I.Kh. Khusnullin, On perturbation of a Schrödinger operATOR ON AXIS BY NARROW POTENTIALS.

(c) Bikmetov A.R., Vil'danova V.F., Khusnullin I.Kh. 2015.

The first author was supported by RFBR (project no. 14-01-97024-r_povolzhe_a). The third author was supported in the framework of basic part of state task in the scope of scientific activity of Ministry of Education and Science of Russia.

Submitted August 18, 2015. 
obeys the conditions $\int_{\mathbb{R}}|W(x)|(1+|x|) d x<\infty$ and $\int_{\mathbb{R}} W(t) d t \leqslant 0$. It was also proved there that once $\int_{\mathbb{R}} W(t) d t>0$, there is no such eigenvalue.

In [7] the operator $\mathcal{H}_{0}-\varepsilon \mathcal{L}_{\varepsilon}$ was considered, where $0<\varepsilon \ll 1, \mathcal{L}_{\varepsilon}: W_{2, l o c}^{2}(\mathbb{R}) \rightarrow L_{2}(\mathbb{R} ; Q)$ is an arbitrary linear operator satisfying uniform in $\varepsilon$ inequality

$$
\left\|\mathcal{L}_{\varepsilon} u\right\|_{L_{2}(\mathbb{R})} \leqslant C\|u\|_{W_{2}^{2}(Q)}
$$

for a finite interval $Q$ in the axis; constant $C$ is independent of $\varepsilon, L_{2}(\mathbb{R} ; Q)$ is the subset of functions $L_{2}(\mathbb{R})$ with supports in $\bar{Q}, W_{2, l o c}^{2}(\mathbb{R})$ is the set of functions defined on space $\mathbb{R}$, whose restriction on each bounded set $D \subset \mathbb{R}$ belongs to $W_{2}^{2}(D)$. Sufficient conditions for the existence of small eigenvalues of the considered operator were obtained. In the case of existence, the asymptotics for the eigenvalues were constructed.

In [8], the effect of the emergence of eigenvalues from a threshold of the essential spectrum was studied for operators with narrow potentials. A particular case of such operator was operator (2). On the basis of the results obtained in [7], there were found sufficient conditions ensuring the emergence of eigenvalues from the threshold of continuous spectrum under the assumption that there exists a number $\gamma>0$ such that $\mu^{-1} \varepsilon^{1 / 2}=o\left(\varepsilon^{\gamma}\right)$.

On the other hand, in view of the results obtained in [9], [10, it is natural to expect that the results of [8] on perturbing discrete spectrum of Schrödginer operators with narrow potentials are valid for a wider class of parameters $\varepsilon, \mu$ satisfying condition (1). In the present work we employ the approach proposed in [11] to show that this conjecture is true and we establish sufficient conditions for the emergence of the eigenvalues from the threshold of the essential spectrum under condition (1).

Suppose that segment $Q=[a, b]$ is such that $x_{j} \in Q$ and $\operatorname{supp} V_{j}(x) \subset Q, j=1,2$. We denote

$$
\langle g\rangle:=\int_{\mathbb{R}} g(t) d t
$$

Our main result is the following theorem.

Theorem 1. Suppose that condition (1) holds true. If

$$
\operatorname{Re}\left(\left\langle V_{1}\right\rangle+\left\langle V_{2}\right\rangle\right)>0,
$$

then there exists the unique eigenvalue $\lambda_{\varepsilon, \mu}$ of operator $\mathcal{H}_{\varepsilon, \mu}$ tending to zero as $\varepsilon \rightarrow 0$. This eigenvalue is simple and its asymptotics reads as

$$
\lambda_{\varepsilon, \mu}=-\frac{1}{4}\left(\varepsilon \mu^{-1}\right)^{2}\left(\left\langle V_{1}\right\rangle+\left\langle V_{2}\right\rangle\right)^{2}+O\left(\varepsilon^{3} \mu^{-3}\right) .
$$

If

$$
\operatorname{Re}\left(\left\langle V_{1}\right\rangle+\left\langle V_{2}\right\rangle\right)<0
$$

then operator $\mathcal{H}_{\varepsilon, \mu}$ has no eigenvalues converging to zero as $\varepsilon \rightarrow 0$.

\section{Auxiliary statements}

It is easy to see that the function

$$
W_{j}(\xi)=\frac{1}{2} \int_{\mathbb{R}}|\xi-t| V_{j}(t) d t
$$

solves the equation

$$
W_{j}^{\prime \prime}(\xi)=V_{j}(\xi), \quad j=1,2 .
$$

Since $x_{1}<x_{2}$, then $\operatorname{supp} V_{1}\left(\frac{x-x_{1}}{\varepsilon}\right) \cap \operatorname{supp} V_{2}\left(\frac{x-x_{2}}{\varepsilon}\right)=\varnothing$ for sufficiently small $\varepsilon$. This is why there exist fixed intervals $Q_{1} \subset Q$ and $Q_{2} \subset Q$ such that $\operatorname{supp} V_{j}\left(\frac{x-x_{j}}{\varepsilon}\right) \subset Q_{j}, j=1,2$ and $Q_{1} \cap Q_{2}=\varnothing$. We choose cut-off functions $\chi_{j}(x)$ satisfying the following conditions: functions $\chi_{j}(x)$ are equal to one as $x \in Q_{j}$, respectively, vanish as $x \notin Q_{j}$ and $\chi_{1}(x) \chi_{2}(x)=0$. 
We follow the approach proposed [11]. We let

$$
\varphi_{\varepsilon, \mu}(x):=1+\varepsilon^{2} \mu^{-1}\left(\chi_{1}(x) W_{1}\left(\frac{x-x_{1}}{\varepsilon}\right)+\chi_{2}(x) W_{2}\left(\frac{x-x_{2}}{\varepsilon}\right)\right) .
$$

We denote by $U_{\varepsilon, \mu}$ the operator of multiplication by function $\varphi_{\varepsilon, \mu}(x)$ :

$$
U_{\varepsilon, \mu}[v]:=\varphi_{\varepsilon, \mu}(x) v
$$

Operator $U_{\varepsilon, \mu}$ makes one-to-one correspondence of $L_{2}(\mathbb{R})$ onto itself. Hence, eigenvalues of operator $\mathcal{H}_{\varepsilon, \mu}$ coincides with the eigenvalues of operator $U_{\varepsilon, \mu}^{-1} \mathcal{H}_{\varepsilon, \mu} U_{\varepsilon, \mu}$.

Lemma 1. Suppose that condition (1) is satisfied. Then operator $U_{\varepsilon, \mu}$ satisfy the estimates

$$
\begin{gathered}
\left|U_{\varepsilon, \mu}^{-1}[1]\right| \leqslant C_{1}, \quad x \in Q, \\
U_{\varepsilon, \mu}^{-1}[1]=1+O\left(\varepsilon \mu^{-1}\right), \quad x \in Q .
\end{gathered}
$$

Proof. Estimate (10) is implied immediately by the definition of functions $\chi_{j}, W_{j}$ and (1), (9). It follows from (9) that

$$
U_{\varepsilon, \mu}^{-1}[1]=\frac{1}{\varphi_{\varepsilon, \mu}(x)}:=\widetilde{\varphi}_{\varepsilon, \mu}(x) .
$$

We expand function $\widetilde{\varphi}_{\varepsilon, \mu}(x)$ into a series in the vicinity of zero:

$$
\widetilde{\varphi}_{\varepsilon, \mu}(x)=\widetilde{\varphi}_{\varepsilon, \mu}(0)+\widetilde{\varphi}_{\varepsilon, \mu}^{\prime}(c) x, \quad 0<c<x, \quad x \in Q,
$$

where

$$
\widetilde{\varphi}_{\varepsilon, \mu}^{\prime}(x)=-\frac{\varepsilon \mu^{-1}}{\varphi_{\varepsilon, \mu}^{2}(x)}\left(\chi_{1}(x) W_{1}^{\prime}\left(\xi_{1}\right)+\chi_{2}(x) W_{2}^{\prime}\left(\xi_{2}\right)+\varepsilon\left(\chi_{1}^{\prime}(x) W_{1}\left(\xi_{1}\right)+\chi_{2}^{\prime}(x) W_{2}\left(\xi_{2}\right)\right)\right),
$$

$\xi_{j}=\left(x-x_{j}\right) \varepsilon^{-1}$.

The definition of function $\widetilde{\varphi}_{\varepsilon, \mu}(x)$ yields

$$
\begin{aligned}
\widetilde{\varphi}_{\varepsilon, \mu}(0)= & \frac{1}{1+q}, \\
\widetilde{\varphi}_{\varepsilon, \mu}^{\prime}(c)= & -\frac{\varepsilon \mu^{-1}}{\varphi_{\varepsilon, \mu}^{2}(c)}\left(\chi_{1}(c) W_{1}^{\prime}\left(\frac{c-x_{1}}{\varepsilon}\right)+\chi_{2}(c) W_{2}^{\prime}\left(\frac{c-x_{2}}{\varepsilon}\right)\right) \\
& -\frac{\varepsilon^{2} \mu^{-1}}{\varphi_{\varepsilon, \mu}^{2}(c)}\left(\chi_{1}^{\prime}(c) W_{1}\left(\frac{c-x_{1}}{\varepsilon}\right)+\chi_{2}^{\prime}(c) W_{2}\left(\frac{c-x_{2}}{\varepsilon}\right)\right),
\end{aligned}
$$

where

$$
q=\varepsilon^{2} \mu^{-1}\left(\chi_{1}(0) W_{1}\left(\frac{-x_{1}}{\varepsilon}\right)+\chi_{2}(0) W_{2}\left(\frac{-x_{2}}{\varepsilon}\right)\right) .
$$

By (1), (8) and the definition of functions $\chi_{j}, W_{j}$ we obtain the estimates

$$
|q|<1, \quad\left|\varphi_{\varepsilon, \mu}^{-2}(c)\right| \leqslant C_{2} .
$$

These estimates and 12 yield

$$
\widetilde{\varphi}_{\varepsilon, \mu}(0)=1+O(q)=1+O\left(\varepsilon^{2} \mu^{-1}\right), \quad \widetilde{\varphi}_{\varepsilon, \mu}^{\prime}(c)=O\left(\varepsilon \mu^{-1}\right) .
$$

The latter estimates imply (11). The proof is complete.

Lemma 2. Suppose that condition (1) is satisfied. Then the representation

$$
U_{\varepsilon, \mu}^{-1} \mathcal{H}_{\varepsilon, \mu} U_{\varepsilon, \mu}=\mathcal{H}_{0}+\varepsilon \mu^{-1} \mathcal{L}_{\varepsilon, \mu}
$$

holds true, where $\mathcal{L}_{\varepsilon, \mu}$ is a second order differential operator with bounded compactly supported coefficients satisfying the estimate

$$
\left\|\mathcal{L}_{\varepsilon, \mu} u\right\|_{L_{2}(\mathbb{R})} \leqslant C_{3}\|u\|_{W_{2}^{2}(Q)},
$$

constant $C_{3}$ is independent of $\varepsilon$. 
Proof. By (2) and (9) we get

$$
\begin{aligned}
\mathcal{H}_{\varepsilon, \mu} U_{\varepsilon, \mu}= & \left(\mathcal{H}_{0}+\mu^{-1}\left(V_{1}\left(\xi_{1}\right)+V_{2}\left(\xi_{2}\right)\right)\right)\left[1+\varepsilon^{2} \mu^{-1}\left(\chi_{1}(x) W_{1}\left(\xi_{1}\right)+\chi_{2}(x) W_{2}\left(\xi_{2}\right)\right)\right] \\
= & U_{\varepsilon, \mu}[1] \mathcal{H}_{0}+\mu^{-1}\left(V_{1}\left(\xi_{1}\right)+V_{2}\left(\xi_{2}\right)\right)+\varepsilon^{2} \mu^{-1}\left(\chi_{1}(x) \mathcal{H}_{0}\left[W_{1}\left(\xi_{1}\right)\right]+\chi_{2}(x) \mathcal{H}_{0}\left[W_{2}\left(\xi_{2}\right)\right]\right) \\
& +\varepsilon^{2} \mu^{-1} \sum_{j=1}^{2}\left(W_{j}\left(\xi_{j}\right) \mathcal{H}_{0}\left[\chi_{j}(x)\right]-2 \frac{d}{d x}\left[\chi_{j}(x)\right] \frac{d}{d x}\left[W_{j}\left(\xi_{j}\right)\right]\right. \\
& \left.\quad-2 W_{j}\left(\xi_{j}\right) \frac{d}{d x}\left[\chi_{j}(x)\right] \frac{d}{d x}-2 \chi_{j}(x) \frac{d}{d x}\left[W_{j}\left(\xi_{j}\right)\right] \frac{d}{d x}\right) \\
& +\varepsilon^{2} \mu^{-2}\left(V_{1}\left(\xi_{1}\right) W_{1}\left(\xi_{1}\right)+V_{2}\left(\xi_{2}\right) W_{2}\left(\xi_{2}\right)+V_{1}\left(\xi_{1}\right) \chi_{2}(x) W_{2}\left(\xi_{2}\right)+V_{2}\left(\xi_{2}\right) \chi_{1}(x) W_{1}\left(\xi_{1}\right)\right) .
\end{aligned}
$$

By (7), the definition of operator $\mathcal{H}_{0}$ and function $\chi_{j}$ it implies

$$
\begin{aligned}
\mu^{-1} & \left(V_{1}\left(\xi_{1}\right)+V_{2}\left(\xi_{2}\right)\right)+\varepsilon^{2} \mu^{-1}\left(\chi_{1}(x) \mathcal{H}_{0}\left[W_{1}\left(\xi_{1}\right)\right]+\chi_{2}(x) \mathcal{H}_{0}\left[W_{2}\left(\xi_{2}\right)\right]\right) \\
& =\mu^{-1}\left(V_{1}\left(\xi_{1}\right)+V_{2}\left(\xi_{2}\right)\right)+\varepsilon^{2} \mu^{-1}\left(\mathcal{H}_{0}\left[W_{1}\left(\xi_{1}\right)\right]+\mathcal{H}_{0}\left[W_{2}\left(\xi_{2}\right)\right]\right) \\
& =\mu^{-1}\left(V_{1}\left(\xi_{1}\right)+V_{2}\left(\xi_{2}\right)\right)-\mu^{-1}\left(V_{1}\left(\xi_{1}\right)+V_{2}\left(\xi_{2}\right)\right)=0 .
\end{aligned}
$$

It follows from the definition of $\chi_{j}$ that

$$
V_{1}\left(\xi_{1}\right) \chi_{2}(x) W_{2}\left(\xi_{2}\right)=0, \quad V_{2}\left(\xi_{2}\right) \chi_{1}(x) W_{1}\left(\xi_{1}\right)=0 .
$$

In view of these identities we obtain that

$$
\begin{aligned}
\mathcal{H}_{\varepsilon, \mu} U_{\varepsilon, \mu}= & U_{\varepsilon, \mu}[1] \mathcal{H}_{0}+\varepsilon^{2} \mu^{-1} \sum_{j=1}^{2}\left(W_{j}\left(\xi_{j}\right) \mathcal{H}_{0}\left[\chi_{j}(x)\right]-2 \frac{d}{d x}\left[\chi_{j}(x)\right] \frac{d}{d x}\left[W_{j}\left(\xi_{j}\right)\right]\right. \\
& \left.-2 W_{j}\left(\xi_{j}\right) \frac{d}{d x}\left[\chi_{j}(x)\right] \frac{d}{d x}-2 \chi_{j}(x) \frac{d}{d x}\left[W_{j}\left(\xi_{j}\right)\right] \frac{d}{d x}\right) \\
& +\varepsilon^{2} \mu^{-2}\left(V_{1}\left(\xi_{1}\right) W_{1}\left(\xi_{1}\right)+V_{2}\left(\xi_{2}\right) W_{2}\left(\xi_{2}\right)\right) .
\end{aligned}
$$

This identity and (9) lead us to identity (13), where

$$
\begin{aligned}
\mathcal{L}_{\varepsilon, \mu}= & U_{\varepsilon, \mu}^{-1}[1] \varepsilon \sum_{j=1}^{2}\left(W_{j}\left(\xi_{j}\right) \mathcal{H}_{0}\left[\chi_{j}(x)\right]-2 \frac{d}{d x}\left[\chi_{j}(x)\right] \frac{d}{d x}\left[W_{j}\left(\xi_{j}\right)\right]-\right. \\
& \left.-2 W_{j}\left(\xi_{j}\right) \frac{d}{d x}\left[\chi_{j}(x)\right] \frac{d}{d x}-2 \chi_{j}(x) \frac{d}{d x}\left[W_{j}\left(\xi_{j}\right)\right] \frac{d}{d x}\right)+ \\
& +U_{\varepsilon, \mu}^{-1}[1] \varepsilon \mu^{-1}\left(V_{1}\left(\xi_{1}\right) W_{1}\left(\xi_{1}\right)+V_{2}\left(\xi_{2}\right) W_{2}\left(\xi_{2}\right)\right) .
\end{aligned}
$$

Let us prove that operator $\mathcal{L}_{\varepsilon, \mu}$ satisfies estimate (14). It follows from (10) that

$$
\begin{aligned}
\left\|\mathcal{L}_{\varepsilon, \mu} u\right\|_{L_{2}(\mathbb{R})} \leqslant & \varepsilon C_{1} \sum_{j=1}^{2}\left(\left\|W_{j}\left(\xi_{j}\right) \mathcal{H}_{0}\left[\chi_{j}(x)\right] u\right\|_{L_{2}(\mathbb{R})}+2\left\|\frac{d}{d x}\left[\chi_{j}(x)\right] \frac{d}{d x}\left[W_{j}\left(\xi_{j}\right)\right] u\right\|_{L_{2}(\mathbb{R})}+\right. \\
& \left.+2\left\|W_{j}\left(\xi_{j}\right) \frac{d}{d x}\left[\chi_{j}(x)\right] \frac{d u}{d x}\right\|\left\|_{L_{2}(\mathbb{R})}+2\right\| \chi_{j}(x) \frac{d}{d x}\left[W_{j}\left(\xi_{j}\right)\right] \frac{d u}{d x} \|_{L_{2}(\mathbb{R})}\right)+ \\
& +\varepsilon \mu^{-1} C_{1}\left(\left\|V_{1}\left(\xi_{1}\right) W_{1}\left(\xi_{1}\right) u\right\|_{L_{2}(\mathbb{R})}+\left\|V_{2}\left(\xi_{2}\right) W_{2}\left(\xi_{2}\right) u\right\|_{L_{2}(\mathbb{R})}\right) .
\end{aligned}
$$

Now we estimate each term in the right hand side of the latter inequality. By the definition of functions $\chi_{j}$ we have

$$
\left\|W_{j}\left(\xi_{j}\right) \mathcal{H}_{0}\left[\chi_{j}(x)\right] u\right\|_{L_{2}(\mathbb{R})}=\left\|W_{j}\left(\xi_{j}\right) \chi_{j}^{\prime \prime}(x) u\right\|_{L_{2}(Q)} \leqslant C_{4}^{j}\|u\|_{L_{2}(Q)} \leqslant C_{4}^{j}\|u\|_{W_{2}^{2}(Q)},
$$


where $C_{4}^{j}=\max _{x \in Q}\left(\left|W_{j}\left(\xi_{j}\right) \chi_{j}^{\prime \prime}(x)\right|\right)$,

$$
\left\|\frac{d}{d x}\left[\chi_{j}(x)\right] \frac{d}{d x}\left[W_{j}\left(\xi_{j}\right)\right] u\right\|_{L_{2}(\mathbb{R})}=\varepsilon^{-1}\left\|\chi_{j}^{\prime}(x) W_{j}^{\prime}\left(\xi_{j}\right) u\right\|_{L_{2}(Q)} \leqslant \varepsilon^{-1} C_{5}^{j}\|u\|_{L_{2}(Q)} \leqslant \varepsilon^{-1} C_{5}^{j}\|u\|_{W_{2}^{2}(Q)},
$$

where $C_{5}^{j}=\max _{x \in Q}\left(\left|\chi_{j}^{\prime}(x) W_{j}^{\prime}\left(\xi_{j}\right)\right|\right)$,

$$
\left\|W_{j}\left(\xi_{j}\right) \frac{d}{d x}\left[\chi_{j}(x)\right] \frac{d u}{d x}\right\|_{L_{2}(\mathbb{R})}=\left\|W_{j}\left(\xi_{j}\right) \chi_{j}^{\prime}(x) \frac{d u}{d x}\right\|_{L_{2}(Q)} \leqslant C_{6}^{j}\left\|\frac{d u}{d x}\right\|_{L_{2}(Q)} \leqslant C_{6}^{j}\|u\|_{W_{2}^{2}(Q)}
$$

where $C_{6}^{j}=\max _{x \in Q}\left(\left|W_{j}\left(\xi_{j}\right) \chi_{j}^{\prime}(x)\right|\right)$,

$$
\left\|\chi_{j}(x) \frac{d}{d x}\left[W_{j}\left(\xi_{j}\right)\right] \frac{d u}{d x}\right\|_{L_{2}(\mathbb{R})}=\varepsilon^{-1}\left\|\chi_{j}(x) W_{j}^{\prime}\left(\xi_{j}\right) \frac{d u}{d x}\right\|_{L_{2}(Q)} \leqslant \varepsilon^{-1} C_{7}^{j}\left\|\frac{d u}{d x}\right\|_{L_{2}(Q)} \leqslant \varepsilon^{-1} C_{7}^{j}\|u\|_{W_{2}^{2}(Q)},
$$

where $C_{7}^{j}=\max _{x \in Q}\left(\left|\chi_{j}(x) W_{j}^{\prime}\left(\xi_{j}\right)\right|\right)$,

$$
\left\|V_{j}\left(\xi_{j}\right) W_{j}\left(\xi_{j}\right) u\right\|_{L_{2}(\mathbb{R})}=\left\|V_{j}\left(\xi_{j}\right) W_{j}\left(\xi_{j}\right) u\right\|_{L_{2}(Q)} \leqslant C_{8}^{j}\|u\|_{L_{2}(Q)} \leqslant C_{8}^{j}\|u\|_{W_{2}^{2}(Q)},
$$

where $C_{8}^{j}=\max _{x \in Q}\left(\left|V_{j}\left(\xi_{j}\right) W_{j}\left(\xi_{j}\right)\right|\right)$. These estimate and 16 imply 14 .

\section{Proof of THE THEOREM}

We introduce the notations

$$
\begin{aligned}
m_{\varepsilon, \mu}^{(1)} & :=\int_{\mathbb{R}} \mathcal{L}_{\varepsilon, \mu}[1] d x, \quad m_{\varepsilon, \mu}^{(2)}:=\int_{\mathbb{R}} \mathcal{L}_{\varepsilon, \mu}\left[\int_{\mathbb{R}}|x-t| \mathcal{L}_{\varepsilon, \mu}[1] d t\right] d x, \\
k_{\varepsilon, \mu} & :=\frac{\varepsilon \mu^{-1}}{2} m_{\varepsilon, \mu}^{(1)}+\frac{\left(\varepsilon \mu^{-1}\right)^{2}}{2} m_{\varepsilon, \mu}^{(2)} .
\end{aligned}
$$

Since operator $\mathcal{L}_{\varepsilon, \mu}$ satisfies inequality (14), Theorem 1 in work [7] implies that once

$$
k_{\varepsilon, \mu}=\varepsilon \mu^{-1} c_{1}+\left(\varepsilon \mu^{-1}\right)^{2} c_{2}+O\left(\left(\varepsilon \mu^{-1}\right)^{3}\right), \quad c_{1}, c_{2}=\text { const },
$$

a sufficient condition for the existence of an eigenvalue converging to zero as $\varepsilon \rightarrow 0$ of the operator $\left(\mathcal{H}_{0}-\varepsilon \mu^{-1} \mathcal{L}_{\varepsilon, \mu}\right)$ is the inequality

$$
\operatorname{Re}\left(c_{1}+\varepsilon \mu^{-1} c_{2}\right)<0
$$

while a sufficient condition for the absence of such eigenvalue is the inequality

$$
\operatorname{Re}\left(c_{1}+\varepsilon \mu^{-1} c_{2}\right)>0 .
$$

If 190 is satisfied, then operator $\left(\mathcal{H}_{0}-\varepsilon \mu^{-1} \mathcal{L}_{\varepsilon, \mu}\right)$ has the unique eigenvalue converging to zero. This eigenvalue is simple and has the asymptotics

$$
\lambda_{\varepsilon, \mu}=-\left(\varepsilon \mu^{-1} c_{1}+\left(\varepsilon \mu^{-1}\right)^{2} c_{2}\right)^{2}+O\left(c_{1}\left(\varepsilon \mu^{-1}\right)^{4}+\left(\varepsilon \mu^{-1}\right)^{5}\right) .
$$

It follows from that

$$
\begin{aligned}
\int_{\mathbb{R}} \mathcal{L}_{\varepsilon, \mu}[1] d x= & \varepsilon \int_{\mathbb{R}} U_{\varepsilon, \mu}^{-1}[1] \sum_{j=1}^{2}\left(W_{j}\left(\xi_{j}\right) \mathcal{H}_{0}\left[\chi_{j}(x)\right]-2 \frac{d}{d x}\left[\chi_{j}(x)\right] \frac{d}{d x}\left[W_{j}\left(\xi_{j}\right)\right]\right) d x \\
& +\varepsilon \mu^{-1} \int_{\mathbb{R}} U_{\varepsilon, \mu}^{-1}[1]\left(V_{1}\left(\xi_{1}\right) W_{1}\left(\xi_{1}\right)+V_{2}\left(\xi_{2}\right) W_{2}\left(\xi_{2}\right)\right) d x
\end{aligned}
$$

Changing the variable, we obtain the estimate

$$
\int_{\mathbb{R}} V_{j}\left(\frac{x-x_{j}}{\varepsilon}\right) W_{j}\left(\frac{x-x_{j}}{\varepsilon}\right) d x=\varepsilon \int_{\mathbb{R}} V_{j}(t) W_{j}(t) d t=O(\varepsilon) .
$$


This estimate and (11) yield

$$
\varepsilon \mu^{-1} \int_{\mathbb{R}} U_{\varepsilon, \mu}^{-1}[1]\left(V_{1}\left(\xi_{1}\right) W_{1}\left(\xi_{1}\right)+V_{2}\left(\xi_{2}\right) W_{2}\left(\xi_{2}\right)\right) d x=O\left(\varepsilon^{2} \mu^{-1}\right) .
$$

The definition of operator $\mathcal{H}_{0}$ and 22) imply

$$
\begin{gathered}
\varepsilon \int_{\mathbb{R}}\left(W_{j}\left(\xi_{j}\right) \mathcal{H}_{0}\left[\chi_{j}(x)\right]-2 \frac{d}{d x}\left[\chi_{j}(x)\right] \frac{d}{d x}\left[W_{j}\left(\xi_{j}\right)\right]\right) d x \\
=\varepsilon \int_{\mathbb{R}}\left(-W_{j}\left(\xi_{j}\right) \chi_{j}^{\prime \prime}(x)-2 \varepsilon^{-1} \chi_{j}^{\prime}(x) W_{j}^{\prime}\left(\xi_{j}\right)\right) d x
\end{gathered}
$$

Integrating twice by parts, by the latter identity, (7), the definition of functions $\chi_{j}(x)$ we obtain

$$
\begin{gathered}
\varepsilon \int_{\mathbb{R}}\left(-W_{j}\left(\xi_{j}\right) \chi_{j}^{\prime \prime}(x)-2 \varepsilon^{-1} \chi_{j}^{\prime}(x) W_{j}^{\prime}\left(\xi_{j}\right)\right) d x=-\int_{\mathbb{R}} \chi_{j}^{\prime}(x) W_{j}^{\prime}\left(\xi_{j}\right) d x \\
=\varepsilon^{-1} \int_{\mathbb{R}} \chi_{j}(x) W_{j}^{\prime \prime}\left(\xi_{j}\right) d x=\varepsilon^{-1} \int_{\mathbb{R}} V_{j}\left(\xi_{j}\right) d x=\int_{\mathbb{R}} V_{j}(t) d t .
\end{gathered}
$$

It follows from (22), (11), (23) and (24) that

$$
\int_{\mathbb{R}} \mathcal{L}_{\varepsilon, \mu}[1] d x=\left\langle V_{1}\right\rangle+\left\langle V_{2}\right\rangle+O\left(\varepsilon \mu^{-1}\right) .
$$

Let us prove the estimate

$$
m_{\varepsilon, \mu}^{(2)}=O(1)
$$

By (17) we get

$$
m_{\varepsilon, \mu}^{(2)}=\int_{\mathbb{R}} \mathcal{L}_{\varepsilon, \mu}\left[\int_{\mathbb{R}}|x-t| \mathcal{L}_{\varepsilon, \mu}[1] d t\right] d x=\int_{\mathbb{R}} \mathcal{L}_{\varepsilon, \mu}[f(x)] d x,
$$

where

$$
f(x):=\int_{\mathbb{R}}|x-t| \mathcal{L}_{\varepsilon, \mu}[1] d t .
$$

The definition of operator $\mathcal{L}_{\varepsilon, \mu}$ and (25) yield

$$
\left|\int_{\mathbb{R}}\right| x-t\left|\mathcal{L}_{\varepsilon, \mu}[1] d t\right|=\left|\int_{Q}\right| x-t\left|\mathcal{L}_{\varepsilon, \mu}[1] d t\right| \leqslant C_{Q}\left|\int_{Q} \mathcal{L}_{\varepsilon, \mu}[1] d t\right| \leqslant C_{9}
$$

as $x \in Q$, where $C_{Q}=\max _{x, t \in Q}|x-t|$. Therefore,

$$
|f(x)| \leqslant C_{9}, \quad x \in Q
$$

Estimate (16) yields

$$
\begin{aligned}
\left|\int_{\mathbb{R}} \mathcal{L}_{\varepsilon, \mu}[f(x)] d x\right| \leqslant & \varepsilon C_{1} \sum_{j=1}^{2}\left(\int_{\mathbb{R}}\left|W_{j}\left(\xi_{j}\right) \chi_{j}^{\prime \prime}(x) f(x)\right| d x+2 \varepsilon^{-1} \int_{\mathbb{R}}\left|\chi_{j}^{\prime}(x) W_{j}^{\prime}\left(\xi_{j}\right) f(x)\right| d x+\right. \\
& \left.+2 \int_{\mathbb{R}}\left|W_{j}\left(\xi_{j}\right) \chi_{j}^{\prime}(x) f^{\prime}(x)\right| d x+2 \varepsilon^{-1} \int_{\mathbb{R}}\left|\chi_{j}(x) W_{j}^{\prime}\left(\xi_{j}\right) f^{\prime}(x)\right| d x\right)+ \\
& +\varepsilon \mu^{-1} C_{1}\left(\int_{\mathbb{R}}\left|V_{1}\left(\xi_{1}\right) W_{1}\left(\xi_{1}\right) f(x)\right| d x+\int_{\mathbb{R}}\left|V_{2}\left(\xi_{2}\right) W_{2}\left(\xi_{2}\right) f(x)\right| d x\right) .
\end{aligned}
$$


Since functions $\chi_{j} V_{j}$ are compactly supported, by (28) we arrive at the estimates

$$
\begin{aligned}
& \int_{\mathbb{R}}\left|W_{j}\left(\xi_{j}\right) \chi_{j}^{\prime \prime}(x) f(x)\right| d x=\int_{Q}\left|W_{j}\left(\xi_{j}\right) \chi_{j}^{\prime \prime}(x) f(x)\right| d x \leqslant C_{10}, \\
& \int_{\mathbb{R}}\left|\chi_{j}^{\prime}(x) W_{j}^{\prime}\left(\xi_{j}\right) f(x)\right| d x=\int_{Q}\left|\chi_{j}^{\prime}(x) W_{j}^{\prime}\left(\xi_{j}\right) f(x)\right| d x \leqslant C_{11}, \\
& \int_{\mathbb{R}}\left|V_{j}\left(\xi_{j}\right) W_{j}\left(\xi_{j}\right) f(x)\right| d x=\int_{Q}\left|V_{j}\left(\xi_{j}\right) W_{j}\left(\xi_{j}\right) f(x)\right| d x \leqslant C_{12} .
\end{aligned}
$$

It follows from (27) that

$$
f^{\prime}(x)=\int_{-\infty}^{x} \mathcal{L}_{\varepsilon, \mu}[1] d t+\int_{+\infty}^{x} \mathcal{L}_{\varepsilon, \mu}[1] d t
$$

We denote

$$
f_{\varepsilon}^{(1)}(x):=\chi_{j}(x) W_{j}^{\prime}\left(\xi_{j}\right), \quad f_{\varepsilon}^{(2)}(x):=\chi_{j}^{\prime}(x) W_{j}\left(\xi_{j}\right) .
$$

By (31) the inequality

$$
\int_{\mathbb{R}}\left|f_{\varepsilon}^{(j)}(x) f^{\prime}(x)\right| d x \leqslant \int_{\mathbb{R}}\left|f_{\varepsilon}^{(j)}(x) \int_{-\infty}^{x} \mathcal{L}_{\varepsilon, \mu}[1] d t\right| d x+\int_{\mathbb{R}}\left|f_{\varepsilon}^{(j)}(x) \int_{+\infty}^{x} \mathcal{L}_{\varepsilon, \mu}[1] d t\right| d x
$$

holds true. Switching the integration order in the second integral, we rewrite the latter inequality as

$$
\int_{\mathbb{R}}\left|f_{\varepsilon}^{(j)}(x) f^{\prime}(x)\right| d x \leqslant 2 \int_{\mathbb{R}}\left|f_{\varepsilon}^{(j)}(x) \int_{-\infty}^{x} \mathcal{L}_{\varepsilon, \mu}[1] d t\right| d x .
$$

By (25) we have

$$
\begin{aligned}
\int_{\mathbb{R}}\left|f_{\varepsilon}^{(j)}(x) \int_{-\infty}^{x} \mathcal{L}_{\varepsilon, \mu}[1] d t\right| d x= & \int_{Q}\left|f_{\varepsilon}^{(j)}(x) \int_{-\infty}^{x} \mathcal{L}_{\varepsilon, \mu}[1] d t\right| d x \leqslant \int_{Q}\left|f_{\varepsilon}^{(j)}(x)\right|\left|\int_{-\infty}^{x} \mathcal{L}_{\varepsilon, \mu}[1] d t\right| d x \\
& \leqslant \int_{Q}\left|f_{\varepsilon}^{(j)}(x)\right|\left|\int_{Q} \mathcal{L}_{\varepsilon, \mu}[1] d t\right| d x \leqslant C_{13}^{j}\left|\int_{Q} \mathcal{L}_{\varepsilon, \mu}[1] d t\right| \leqslant C_{14}^{j} .
\end{aligned}
$$

This inequality and 32 implies that

$$
\int_{\mathbb{R}}\left|f_{\varepsilon}^{(j)}(x) f^{\prime}(x)\right| d x \leqslant 2 C_{14}^{j}
$$

This inequality and (29), (30) yield estimate (26).

It follows from (17), (25), (26) that

$$
k_{\varepsilon, \mu}=\frac{\varepsilon \mu^{-1}}{2}\left(\left\langle V_{1}\right\rangle+\left\langle V_{2}\right\rangle\right)+O\left(\varepsilon^{2} \mu^{-2}\right) .
$$

Thus, inequality (18) holds true once

$$
c_{1}=\frac{\left\langle V_{1}\right\rangle+\left\langle V_{2}\right\rangle}{2}, \quad c_{2}=O(1) .
$$

It follows from $(19)$ and $(20)$ that the sufficient condition for the existence of an eigenvalue converging to zero as $\varepsilon \rightarrow 0$ for the operator $\left(\mathcal{H}_{0}+\varepsilon \mu^{-1} \mathcal{L}_{\varepsilon, \mu}\right)$ is the inequality

$$
\operatorname{Re}\left(c_{1}+\varepsilon \mu^{-1} c_{2}\right)>0
$$

while the sufficient condition for the absence of such eigenvalue is the inequality

$$
\operatorname{Re}\left(c_{1}+\varepsilon \mu^{-1} c_{2}\right)<0 .
$$

It follows from (1) and (33) that for sufficiently small $\varepsilon, \mu$ the sign of $\operatorname{Re}\left(c_{1}+\varepsilon \mu^{-1} c_{2}\right)$ coincides with the sign of $\operatorname{Re}\left(c_{1}\right)$. Therefore, by (34), (35) and (33) we arrive at inequalities (4) and (6). Asymptotics (5) is implied by (21) and (33). 


\section{Concluding REMARKS}

It follows from Theorem 1 in work [7] that apart of the eigenvalue converging to zero, all other eigenvalues of operator $\mathcal{H}_{\varepsilon, \mu}$ (if they exist) tend to infinity as $\varepsilon \rightarrow 0$.

Acknowledgments. The authors thank D.I. Borisov for useful remarks and R.R. Gadyl'shin for the attention to the work.

\section{BIBLIOGRAPHY}

1. T. Kato. Perturbation theory for linear operators. Springer, New York (1966).

2. D.I. Borisov, R.R. Gadyl'shin. On spectrum of periodic operator with a small localized perturbation // Izv. AN. Ser. Matem. 72:4, 37-66 (2008). [Izv. Math. 72:4, 659-688 (2008).]

3. B. Simon. The bound state of weakly coupled Schrödinger operators in one and two dimensions // Ann. Phys. 97:2, 279-288 (1976).

4. R. Blankenbecler, M.L. Goldberger, B. Simon. The bound states of weakly coupled long-range one-dimensional quantum Hamiltonians // Ann. Phys. 108:1, 69-78 (1977).

5. M. Klaus. On the bound state of Schrödinger operators in one dimension // Ann. Phys. 108:2, 288-300 (1977).

6. M. Klaus, B. Simon. Coupling constant thresholds in nonrelativistic quantum mechanics. I. Shortrange two-body case // Ann. Phys. 130:2, 251-281 (1980).

7. R.R. Gadyl'shin. Local perturbations of the Schrödinger operator on the axis // Teor. Matem. Fiz. 132:1, 97-104 (2002). [Theor. Math. Phys. 132:1, 976-982 (2002).]

8. R.R. Gadyl'shin, I. Kh. Khusnullin. Schrödinger operator on the axis with potentials depending on two parameters // Alg. Anal. 22:6, 50-66 (2010). [St. Petersburg Math. J. 22:6, 883-894 (2011).]

9. R.R. Gadyl'shin, I.Kh. Khusnullin. Perturbation of the Shrödinger operator by a narrow potential // Ufimskij Matem. Zhurn. 3:3, 55-66 (2011). [Ufa Math. J. 3:3, 54-64 (2011).]

10. I.Kh. Khusnullin. A perturbed boundary eigenvalue problem for the Schrödinger operator on an interval // Zh. Vychisl. Mat. Mat. Fiz. 50:4, 679-698 (2010). [Comp. Math. Math. Phys. 50:4, 646-664 (2010).]

11. D.I. Borisov, R.Kh. Karimov, T.F. Sharapov. Initial length scale estimate for waveguides with some random singular potentials // Ufimskij Matem. Zhurn. 7:2, 35-56 (2015). [Ufa Math. J. 7:2, $33-54(2015)$.]

Bikmetov Aidar Renatovich,

Bashkir State Pedagogical University named after M. Akhmulla,

October rev. st. 3a,

450000, Ufa, Russia

E-mail: BikmetovAR@yandex.ru

Vil'danova Venera Fidarisovna,

Bashkir State Pedagogical University named after M. Akhmulla,

October rev. st. 3a,

450000, Ufa, Russia

E-mail: gilvenera@mail.ru

Khusnullin Il'fat Khamzievich,

Bashkir State Pedagogical University named after M. Akhmulla,

October rev. st. 3a,

450000, Ufa, Russia

E-mail: KhusnullinIKh@mail.ru 